\title{
Theory of Thermally Activated Vortex Bundles Flow Over the Directional- Dependent Potential Barriers in Type-II Superconductors
}

\author{
Wei Yeu Chen ${ }^{*}$ \\ Department of Physics, Tamkang University, Tamsui 25137, Taiwan, China
}

\begin{abstract}
The thermally activated vortex bundle flow over the directional-dependent energy barrier in type-II superconductors is investigated. The coherent oscillation frequency and the mean direction of the random collective pinning force of the vortex bundles are evaluated by applying the random walk theorem. The flow velocity of the vortex bundles is obtained self-consistently. The temperature- and field-dependent Hall and longitudinal resistivities induced by the bundle flow for type-II superconducting bulk materials and thin films are calculated. All the results are in agreement with the experiments. PACS: 74.25.Fy, 74.25.Qt, 74.72.-h.
\end{abstract}

Keywords: Thermally activated bundle flow, directional-dependent potential barrier, vortex dynamics.

\section{INTRODUCTION}

In type-II superconductor [1-24], when the applied magnetic field $B>B_{C_{1}}$, the flux lines penetrate the superconducting sample to form a long-range order of vortex lattice or flux line lattice if the sample is homogeneous [1]. However, the quenched disorder always destroys the long-range order of the vortex lattice for quenched disordered type-II superconductors, after which only short-range order, the vortex bundle, remains [3, 6-11]. The vortex lines inside the vortex bundle oscillate about their equilibrium positions due to thermally agitation for finite temperature $[7,8]$.

In this paper we are going to develop a self-consistent theorem of thermally activated vortex bundles flow over the directional-dependent potential barriers. The coherent frequency of oscillation of the vortex bundle, and the mean direction of the random collective pinning forces of the vortex bundles are evaluated by applying the theorem of random walk. The directional-dependent potential barrier generated by the Magnus force and the random collective pinning force and strong pinning force are calculated. The bundle flow velocity is then obtained self-consistently. Finally, the Hall and longitudinal resistivities induced by the bundle flow are calculated.

The rest of this paper is organized as follows. In section 2 , a mathematical model is presented. In section 3, the coherent frequency of oscillation of the vortex bundles and the mean direction of the random collective pinning forces of the vortex bundles are calculated. The directional-dependent energy barrier is obtained in section 4 . In section 5, the bundle flow velocity is evaluated, the Hall and longitudinal resistivities are calculated. Finally, the concluding remarks are given in section 6 .

*Address correspondence to this author at the Department of Physics, Tamkang University, Tamsui 25137, Taiwan, China; Tel: 886-2-28728682; Fax: 886-2-28728682; E-mail: wychen@mail.tku.edu.tw

\section{MATHEMATICAL DESCRIPTION OF THE MODEL}

Let us consider a type-II conventional or high- $T_{c}$ superconductor, the Hamiltonian of the fluctuation for the flux line lattice (FLL) in the $z$-direction is given by $[9,11,12]$.

$$
H=H_{f}+H_{R}
$$

where $H_{f}=H_{k i n}+H_{e}$ represents the Hamiltonian for the free modes [9, 11, 12], with $H_{k i n}$ the kinetic energy part [9, $11,12]$

$$
H_{k i n}=\frac{1}{2 \rho} \sum_{\vec{K} \mu} P_{\mu}(\vec{K}) P_{\mu}(-\vec{K})
$$

$H_{e}$ the elastic energy part $[9,11,13]$,

$$
\begin{aligned}
H_{e}=\frac{1}{2} \sum_{\vec{K} \mu \nu} C_{L} & K_{\mu} K_{\nu} S_{\mu}(\vec{K}) S_{\nu}(-\vec{K}) \\
& +\frac{1}{2} \sum_{\vec{K} \mu}\left(C_{66} K_{\perp}^{2}+C_{44} K_{z}^{2}\right) S_{\mu}(\vec{K}) S_{\mu}(-\vec{K})
\end{aligned}
$$

and $H_{R}$ represents the random Hamiltonian, given as $[9,11$, 12],

$$
H_{R}=\sum_{\vec{K} \mu} f_{R \mu}(\vec{K}) S_{\mu}(-\vec{K})
$$

where $(\mu, v)=(x, y), \rho$ is the effective mass density of the flux line [14], $K_{\perp}^{2}=K_{x}^{2}+K_{y}^{2}, P_{\mu}(\vec{K}), S_{\mu}(\vec{K})$ are the Fourier transformations of the momentum and displacement operators, and $C_{L}, C_{11}, C_{44}$ and $C_{66}$ are temperature- and $\vec{K}$ dependent bulk modulus, compression modulus, tilt modulus and shear modulus, respectively. $\vec{f}_{R}(\vec{K})$ is the Fourier transformation of the collective pinning force $\vec{f}_{R}(\vec{r})=-\vec{\nabla} V_{R}(\vec{r})$, with $V_{R}(\vec{r})$ the random potential energy of the collective pinning $[15,16]$, which is the sum of the contributions of defects within a distance $\xi$ of the vortex core position $\vec{r}$, 
where $\xi$ is the temperature-dependent coherent length. The correlation functions of the random collective pinning force are assumed to be the short-range correlation [9],

$\overline{<<f_{R \alpha}(\vec{k}) f_{R \beta}^{*}\left(\vec{k}^{\prime}\right)>>_{t h}}=\beta^{C}(T, B) \delta_{\alpha \beta} \delta\left(\vec{k}-\vec{k}^{\prime}\right)$

where $\overline{<<>}_{\text {th }}$ are the quantum, thermal, and random averages, and $\beta^{C}(T, B)$ is the temperature- and magnetic field-dependent correlation strength.

The equation of motion of the displacement operator $S_{\mu}(\vec{K})$ can be obtained from Eq. (1) as

$$
\begin{aligned}
\rho \ddot{S}_{\mu}(\vec{K})+ & C_{L}(\vec{K} \cdot \vec{S}(\vec{K})) K_{\mu} \\
& +\left(C_{66} K_{\perp}^{2}+C_{44} K_{z}^{2}\right) S_{\mu}(\vec{K})+f_{\mu}(\vec{K})=0
\end{aligned}
$$

Then the solution of Eq. (6) can be obtained as

$S_{\mu}(\vec{K})=S_{R \mu}(\vec{K})+S_{f \mu}(\vec{K})$

where $S_{R \mu}(\vec{K})$ denotes the deformation displacement operator of the FLL due to the collective pinning of the random function $\vec{f}_{R \mu}(\vec{K})$, and $S_{f \mu}(\vec{K})$ is the displacement operator for the fluctuation of the free modes. They are given by

$$
\begin{aligned}
S_{R \mu}(\vec{K})=[ & {\left[\left(\vec{K} \cdot \vec{f}_{R}(\vec{K})\right) \frac{\delta_{\alpha, 1}}{K_{\perp}}\right] \cdot \frac{1}{C_{11} K_{\perp}^{2}+C_{44} K_{z}^{2}}+} \\
& {\left[f_{R \alpha}(\vec{K})-\left(\vec{K} \cdot \vec{f}_{R}(\vec{K})\right) \frac{\delta_{\alpha, 1}}{K_{\perp}}\right] \cdot \frac{1}{C_{66} K_{\perp}^{2}+C_{44} K_{z}^{2}} }
\end{aligned}
$$

and

$S_{f \mu}(\vec{K})=\sqrt{\frac{\hbar}{2 \rho \omega_{K \mu}}}\left(\alpha_{-\vec{K} \mu}^{+}+\alpha_{\vec{K} \mu}\right)$

respectively, where $\mu=1$ presents the component parallel to the $\vec{K}_{\perp}$ direction, while $\mu=2$ is perpendicular to the $\vec{K}_{\perp}$ direction. It is understood that the free Hamiltonian can be diagonalized with the eigenmodes spectrum $[9,11,12]$.

$\omega_{K 1}=\left[\frac{1}{\rho}\left(C_{11} K_{\perp}^{2}+C_{44} K_{z}^{2}\right)\right]^{\frac{1}{2}} \omega_{K 2}=\left[\frac{1}{\rho}\left(C_{66} K_{\perp}^{2}+C_{44} K_{z}^{2}\right)\right]^{\frac{1}{2}}$

with $\alpha_{\vec{K} \mu}^{+}, \alpha_{\vec{K} \mu}$ are the creation and the annihilation operators for the corresponding eigenmodes.

The quenched disorder destroys the long-range order of the FLL, after which only short-range order, the vortex bundle, prevails. The corresponding size of vortex bundle $|\vec{R}|$ is determined by the relation $[11,12]$.

$\overline{\left.<<\left|\vec{S}_{R}(\vec{R})-\vec{S}_{R}(0)\right|^{2}>\right\rangle_{t h}}=r_{f}^{2}$

where $r_{f}$ represents the random collective pinning force range.

\section{COHERENT OSCILLATION FREQUENCY AND MEAN DIRECTION OF COLLECTIVE PINNING FORCE FOR VORTEX BUNDLES}

Let us consider a p-type superconductor, with the applied magnetic field $\vec{B}$ in the $\mathrm{z}$-axis and the external current density $\vec{J}$ in the x-axis $\vec{J}=J \vec{e}_{x}$ with $J<J_{C}$, where $J_{C}$ is the critical current density of the superconductor. The equation of motion of the vortex line inside the vortex bundle driving by the thermal radiation of frequency $\omega$ is given by

$$
\frac{d^{2} \vec{r}_{v}}{d t^{2}}=\frac{q \vec{E} e^{i \omega t}}{M_{v}}+\frac{J \Phi_{0}}{M_{v}} \vec{e}_{x} \times \vec{e}_{z}-\left(\frac{1}{\tau_{R}} \frac{d \vec{r}_{v}}{d t}\right)-\left(\frac{k_{R}}{M_{v}} \vec{r}_{v}\right)+\frac{\vec{f}_{e l}}{M_{v}}
$$

where $\vec{r}_{v}$ is the displacement of vortex line from its equilibrium position, $\vec{E}$ denotes the electric field of the thermal radiation, $M_{v}$ stands for the effective mass, and $q$ is the total circulating charge of the vortex line, $\Phi_{0}$ is the unit flux, $1 / \tau_{R}$ characterizes the damping rate associated with the motion of the vortex line, $k_{R}$ represents the restoring force constant for the vortex line under the action of random collective pinning force, and $\vec{f}_{e l}$ is the elastic force of the vortex line inside the vortex bundle. The homogeneous solution of the equation (12) vanishes quickly due to the presence of damping. The particular solution includes two parts: the time-dependent and time-independent parts.

The time-dependent part of the particular solution oscillates with frequency $\omega$ about a new equilibrium position, which is determined by the time-independent part of the particular solution. By identifying the oscillation energy of the vortex line inside the potential barrier with the thermal energy, the thermal oscillation frequency $v$ of the individual vortex inside the potential barrier can be expressed as

$v=\bar{v} \sqrt{T}$

with $\bar{v}=(1 / \pi A) \sqrt{k_{B} / 2 M_{v}}$, where $A$ stands for the random- and thermal-averaged amplitude of the oscillation of the vortex line in the bundle, $k_{B}$ is the Boltzmann constant.

However, the oscillations of vortex lines inside the vortex bundle are not coherent, namely, their oscillations are at random. To obtain the coherent oscillation frequency $\boldsymbol{v}_{c}$ of the vortex bundle as a whole, by utilizing the random walk's theorem, the frequency $v$ in equation (13) must be divided by the square root of $\mathrm{N}$, the number of vortices inside the vortex bundle

$$
v_{c}=\frac{v}{\sqrt{N}}=\frac{\bar{v} \sqrt{T} \sqrt{\Phi_{0}}}{R \sqrt{\pi B}}
$$

where $R$ is the transverse size of the vortex bundle and $B$ is the value of the applied magnetic field. 
The time-independent part of the particular solution is give by

$\vec{r}_{p}=\frac{-J \Phi_{0} \vec{e}_{y}+\vec{f}_{e l}}{k_{R}}$

The above result indicates that the vortex line moves to a new equilibrium position $\vec{r}_{p}$ from its original one. Since the elastic force is much less than the Lorentz force, the angle between the random collective pinning force and the positive $y$-direction measured in the counterclockwise sense can be obtained approximately as

$\theta \cong \frac{\left|\vec{f}_{e l}\right|}{\left|\vec{f}_{L}\right|}=\frac{\left|\vec{f}_{e l}\right|}{J \Phi_{0}}$

where $\left|\vec{f}_{e l}\right|$ and $\left|\vec{f}_{L}\right|$ are the magnitudes of elastic force and Lorentz force of the vortex line, respectively. Taking into account the fact that the compression modulus $C_{11}$ is much larger than shear modulus $C_{66}[9,10]$, we arrive at, the magnitude of the displacement vector $\left|\vec{S}_{f}(\vec{r})\right|$ of the vortex line as well as its corresponding elastic force $\left|\vec{f}_{e l}\right|$ is proportional to $\sqrt{k_{B} / C_{66}}$, or $(1 / \sqrt{B}) \sqrt{T /\left(T_{C}-T\right)}$.

The temperature- and field-dependent $\theta$ can therefore be written as

$\theta(T, B)=\alpha \frac{1}{\sqrt{B}} \sqrt{\frac{T}{T_{c}-T}}$

where $\alpha$ is a proportional constant. By applying the random walk theorem, the mean angle $\Theta(T, B)$ between the random collective pinning force of vortex bundle and positive $y-$ direction measures in counterclockwise sense, can be expressed as

$\Theta(T, B)=\sqrt{N} \theta(T, B)=\bar{\alpha} \sqrt{\frac{T}{T_{c}-T}}$

where $\bar{\alpha}=\alpha R \sqrt{\pi / \Phi_{0}}$.

\section{DIRECTIONAL-DEPENDENT ENERGY BARRIER}

In this section we shall calculate the directionaldependent energy barrier of the vortex bundles generated by the Magnus force, random collective pinning force, and the strong pinning force inside the vortex bundle. The directional-dependent potential barrier means that the energy barrier is a function of direction of the thermally activated motion. Assuming that the external current is in the $\mathrm{x}$ direction and the applied magnetic field in the z-direction, the Magnus force acting on the vortex bundle can then be obtained as

$\vec{F}_{M}=\bar{V} n_{s} e\left(\vec{v}_{T}-\vec{v}_{b}\right) \times B \hat{e}_{z}$

where $\vec{v}_{b}$ is the velocity of the thermally activated vortex bundle flow, $\bar{V}$ is the volume of the vortex bundle, $e$ is the electron charge, $n_{s}$ and $\vec{v}_{T}$ are the supercharge density and its velocity, respectively, with $\vec{J}=n_{s} e \vec{v}_{T}=J \vec{e}_{x}$, and $\vec{B}=B \vec{e}_{z}$. From the theory of mechanics, the potential generated by a force field $\vec{F}(r)$ is

$V(\vec{R})-V(0)=-\int_{0}^{\vec{R}} \vec{F}(r) \cdot d \vec{r}$

After some algebra, the directional-dependent energy barrier of the vortex bundles both in the positive and negative $\mathrm{x}$-direction as well as $\mathrm{y}$-direction are obtained as

$$
\begin{aligned}
& U+\bar{V} R\left(J B \frac{v_{b y}}{v_{T}}-<F_{p_{x}}>_{R}\right) \quad U-\bar{V} R\left(J B \frac{v_{b y}}{v_{T}}-<F_{p_{x}}>_{R}\right) \\
& U+\bar{V} R\left(J B-J B \frac{v_{b x}}{v_{T}}-<F_{p_{y}}>_{R}\right) \\
& U-\bar{V} R\left(J B-J B \frac{v_{b x}}{v_{T}}-<F_{p_{y}}>_{R}\right)
\end{aligned}
$$

respectively, where $U$ is the potential barrier generated by the strong pinning force due to the randomly distributed strong pinning sites inside the vortex bundle, $R$ represents the transverse size of the vortex bundle, the range of $U$ is assumed to be of the order $R$, and $\left\langle\vec{F}_{p}\right\rangle_{R}$ stands for the random average of the random collective pinning force per unit volume.

\section{BUNDLE FLOW VELOCITY AND ITS INDUCED LONGITUDINAL AND HALL RESISTIVITIES}

The results of equation (21) indicate that the directionaldependent potential barrier used for calculating the vortex bundles flow velocity actually itself contains the vortex bundles flow velocity. Therefore, the velocity of thermally activated vortex bundles flow over the directional-dependent energy barrier must be solved self-consistently as follows

$$
\begin{aligned}
& v_{b y}=v_{c} R\left\{\exp \left[\frac{-1}{k_{B} T}\left(U+\bar{V} R\left(J B-J B \frac{v_{b x}}{v_{T}}-<F_{p_{y}}>_{R}\right)\right)\right]\right. \\
& \left.-\exp \left[\frac{-1}{k_{B} T}\left(U-\bar{V} R\left(J B-J B \frac{v_{b x}}{v_{T}}-<F_{p_{y}}>_{R}\right)\right)\right]\right\}
\end{aligned}
$$

$$
\begin{aligned}
& v_{b x}=v_{c} R\left\{\exp \left[\frac{-1}{k_{B} T}\left(U+\bar{V} R\left(J B \frac{v_{b y}}{v_{T}}-<F_{p_{x}}>_{R}\right)\right)\right]\right. \\
& \left.-\exp \left[\frac{-1}{k_{B} T}\left(U-\bar{V} R\left(J B \frac{v_{b y}}{v_{T}}-<F_{p_{x}}>_{R}\right)\right)\right]\right\}
\end{aligned}
$$

with $v_{c}$ is the coherent oscillation frequency of the vortex bundle. Taking into account the fact that $\left(v_{b x} / v_{T}\right)<<1$, the vortex bundle flow velocity can be approximately obtained

$$
\begin{aligned}
& \text { as, } v_{b y}=\left(\frac{\bar{\nu}}{R} \sqrt{\frac{T \Phi_{0}}{\pi B}}\right) R \exp \left(\frac{-U}{k_{B} T}\right)\left\{\operatorname { e x p } \left[\frac{-\bar{V} R}{k_{B} T}\right.\right. \\
& \left.\left(J B-K F_{p}>_{R} \mid \cos \Theta\right)\right] \\
& \left.-\exp \left[\frac{+\bar{V} R}{k_{B} T}\left(J B-\left|<F_{p}>_{R}\right| \cos \Theta\right)\right]\right\}
\end{aligned}
$$




$$
\begin{gathered}
v_{b x}=\left(\frac{\bar{\nu}}{R} \sqrt{\frac{T \Phi_{0}}{\pi B}}\right) R \exp \left(\frac{-U}{k_{B} T}\right)\left\{\operatorname { e x p } \left[\frac { - \overline { V } R } { k _ { B } T } \left(\frac{-J B\left|v_{b y}\right|}{v_{T}}\right.\right.\right. \\
\left.\left.+\left|<F_{p}\right\rangle_{R} \mid \sin \Theta\right)\right] \\
\left.-\exp \left[\frac{+\bar{V} R}{k_{B} T}\left(\frac{-J B\left|v_{b y}\right|}{v_{T}}+K F_{p}>_{R} \mid \sin \Theta\right)\right]\right\}
\end{gathered}
$$

where $\Theta$ is the mean angle between the random collective pinning force of the vortex bundles and positive $y$-direction measured in the counterclockwise sense. By considering the identities $\vec{E}=-\vec{v}_{b} \times \vec{B}, \rho_{x x}=E_{x} / J, \rho_{x y}=E_{y} / J$ together with Eq. (18) and bearing in mind that $\Theta$ is usually very small, the longitudinal and Hall resistivities induced by the vortex bundles flow can now be obtained, respectively, as follows:

$$
\begin{aligned}
& \rho_{x x}=\frac{\bar{v} \sqrt{B T \Phi_{0}}}{J \sqrt{\pi}} \exp \left(\frac{-U}{k_{B} T}\right)\left\{\exp \left[\frac{\bar{V} R}{k_{B} T}\left(J B-\left(\frac{\beta^{C}(T, B)}{\bar{V}}\right)^{\frac{1}{2}}\right)\right]\right. \\
& \left.-\exp \left[\frac{-\bar{V} R}{k_{B} T}\left(J B-\left(\frac{\beta^{C}(T, B)}{\bar{V}}\right)^{\frac{1}{2}}\right)\right]\right\} \\
& \rho_{x y}=\frac{-\bar{\nu} \sqrt{B T \Phi_{0}}}{J \sqrt{\pi}} \exp \left(\frac{-U}{k_{B} T}\right)\left\{\operatorname { e x p } \left[\frac { \overline { V } R } { k _ { B } T } \left(\left(\frac{\beta^{C}(T, B)}{\bar{V}}\right)^{\frac{1}{2}}\right.\right.\right. \\
& -\exp \left[\frac{-\bar{V} R}{k_{B} T}\left(\left(\frac{\beta^{C}(T, B)}{\bar{V}}\right)^{\frac{1}{2}} \bar{\alpha} \sqrt{\frac{T}{T_{C}-T}}-J B \frac{\left|v_{b y}\right|}{v_{T}}\right)\right]
\end{aligned}
$$

and

$\left|v_{b y}\right|=J \rho_{x x} / B$

where $\left(\beta^{C}(T, B) / \bar{V}\right)^{\frac{1}{2}}$ is the magnitude of the random average of the random collective pinning force per unit volume, and $\bar{\alpha}$ is a proportional constant.

In fact, the arguments in the exponential functions inside the curly bracket of Eqs. (26) and (27) are very small when the Lorentz force is close to the random collective pinning force, we finally obtain the temperature- and field-dependent longitudinal and Hall resistivities as

$$
\begin{gathered}
\left.\rho_{x x}=\frac{\bar{v} \sqrt{B \Phi_{0}}}{J \sqrt{\pi T}} \exp \left(\frac{-U}{k_{B} T}\right)\left(\frac{2 \bar{V} R}{k_{B}}\right)\left[J B-\left(\frac{\beta^{C}(T, B)}{\bar{V}}\right)^{\frac{1}{2}}\right)\right] \\
\rho_{x y}=\frac{-\bar{\nu} \sqrt{B \Phi_{0}}}{J \sqrt{\pi T}} \exp \left(\frac{-U}{k_{B} T}\right)\left(\frac{2 \bar{V} R}{k_{B}}\right)\left[\left(\frac{\beta^{C}(T, B)}{\bar{V}}\right)^{\frac{1}{2}}\right. \\
\left.\bar{\alpha} \sqrt{\frac{T}{T_{C}-T}}-J B \frac{\left|v_{b y}\right|}{v_{T}}\right]
\end{gathered}
$$

respectively, with $\left|v_{b y}\right|=J \rho_{x x} / B$.
In the following subsections, we shall calculate the longitudinal and Hall resistivities induced by thermally activated vortex bundles flow for type-II superconducting bulk materials and thin films as functions of temperature and applied magnetic field. The results are then comparing with experiments.

\subsection{Induced Longitudinal and Hall Resistivities for Type-II Superconducting Bulk Materials}

Now let us concentrate on the case for type-II superconducting bulk materials, the volume $\bar{V}$ for the vortex bundle in Eqs. (29) and (30) is given as $\bar{V}=\pi R^{2} L$, where $R(L)$ is the transverse (longitudinal) size of the vortex bundle. In this case, the longitudinal and Hall resistivities for type-II superconducting bulk materials now become

$$
\begin{gathered}
\rho_{x x}=\frac{\bar{v} \sqrt{\Phi_{0}} \sqrt{B}}{J \sqrt{\pi} \sqrt{T}} \exp \left(\frac{-U}{k_{B} T}\right)\left[\frac{2 \pi R^{3} L}{k_{B}}\right]\left[J B-\left(\frac{\beta^{C}(T, B)}{\bar{V}}\right)^{\frac{1}{2}}\right] \\
\rho_{x y}=\frac{-\bar{\nu} \sqrt{\Phi_{0}} \sqrt{B}}{J \sqrt{\pi} \sqrt{T}} \exp \left(\frac{-U}{k_{B} T}\right)\left[\frac{2 \pi R^{3} L}{k_{B}}\right]\left[\left(\frac{\beta^{C}(T, B)}{\bar{V}}\right)^{\frac{1}{2}}\right. \\
\left.\bar{\alpha} \sqrt{\frac{T}{T_{c}-T}}-J B \frac{\left|v_{b y}\right|}{v_{T}}\right]
\end{gathered}
$$

respectively, with $\left|v_{b y}\right|=J \rho_{x x} / B$.

\section{1.a. Longitudinal and Hall Resistivities for Constant Temperature}

Under the framework of present theory, the results of numerical calculations for $\rho_{x x}$ and $\rho_{x y}$, when the temperature is kept at $T=91 \mathrm{~K}$, are given in Table 1. It is shown that as the applied magnetic field decreasing, $\rho_{x y}$ initially decreases, crossing over from positive to negative near 3.03 Tesla, after reaching a minimum at 1 Tesla, then increases again; while $\rho_{x x}$ decreases monotonically. These results are in agreement with the experimental plotting for $\rho_{x y}$ and $\rho_{x x}$ versus applied magnetic field on $\mathrm{YBa}_{2} \mathrm{Cu}_{3} \mathrm{O}_{7-\delta}$ high- $T_{c}$ bulk materials [17]. In obtaining the above results, the following approximate data have been employed:

$$
\begin{aligned}
& R=2 \times 10^{-8} \mathrm{~m}, L=10^{-6} \mathrm{~m}, \\
& J=10^{6} \mathrm{~A} / \mathrm{m}^{2}, T_{c}=92 \mathrm{~K}, v_{T}=10^{3} \mathrm{~m} / \mathrm{sec}, \bar{v}=10^{11} \mathrm{sec}^{-1}, \\
& \bar{\alpha}=5.59 \times 10^{-5} T^{-1 / 2}, \exp \left(-U / k_{B} T\right)=2.07 \times 10^{-2} \mathrm{~m}, \\
& \left(\beta^{C}(B=3.5) / \bar{V}\right)^{1 / 2}=3.4506 \times 10^{6} \mathrm{~N} / \mathrm{m}^{3}, \\
& \left(\beta^{C}(3.03) / \bar{V}\right)^{1 / 2}=2.9849 \times 10^{6} \mathrm{~N} / \mathrm{m}^{3}, \\
& \left(\beta^{C}(2.5) / \bar{V}\right)^{1 / 2}=2.4605 \times 10^{6} \mathrm{~N} / \mathrm{m}^{3}, \\
& \left(\beta^{C}(2) / \bar{V}\right)^{1 / 2}=1.9668 \times 10^{6} \mathrm{~N} / \mathrm{m}^{3}, \\
& \left(\beta^{C}(1.5) / \bar{V}\right)^{1 / 2}=1.4748 \times 10^{6} \mathrm{~N} / \mathrm{m}^{3},
\end{aligned}
$$




$$
\begin{aligned}
& \left(\beta^{C}(1) / \bar{V}\right)^{1 / 2}=9.854 \times 10^{5} \mathrm{~N} / \mathrm{m}^{3}, \\
& \left(\beta^{C}(0.75) / \bar{V}\right)^{1 / 2}=7.4042 \times 10^{5} \mathrm{~N} / \mathrm{m}^{3},
\end{aligned}
$$$$
\text { and }\left(\beta^{C}(0.5) / \bar{V}\right)^{1 / 2}=4.915 \times 10^{5} \mathrm{~N} / \mathrm{m}^{3} \text {. }
$$

Table 1. $\rho_{x y}$ and $\rho_{x x}$ versus Applied Magnetic Field in Tesla for $\mathrm{YBa}_{2} \mathrm{Cu}_{3} \mathrm{O}_{7-\delta} \mathrm{High}-\mathrm{T}_{c}$ Superconducting Bulk Materials at $T=91 \mathrm{~K}$

\begin{tabular}{|c|c|c|}
\hline $\boldsymbol{B}(\boldsymbol{T})$ & $\boldsymbol{\rho}_{x y}(\boldsymbol{\Omega} \boldsymbol{m})$ & $\boldsymbol{\rho}_{x x}(\boldsymbol{\Omega} \boldsymbol{m})$ \\
\hline \hline 3.5 & $1.2914 \times 10^{-9}$ & $1.8739 \times 10^{-6}$ \\
\hline 3.03 & $1.7658 \times 10^{-15}$ & $1.5916 \times 10^{-6}$ \\
\hline 2.5 & $-1.4627 \times 10^{-9}$ & $1.2663 \times 10^{-6}$ \\
\hline 2.0 & $-2.7907 \times 10^{-9}$ & $9.5142 \times 10^{-7}$ \\
\hline 1.5 & $-3.9996 \times 10^{-9}$ & $6.2539 \times 10^{-7}$ \\
\hline 1.0 & $-4.6405 \times 10^{-9}$ & $2.9669 \times 10^{-7}$ \\
\hline 0.75 & $-3.9801 \times 10^{-9}$ & $1.6825 \times 10^{-7}$ \\
\hline 0.5 & $-2.0098 \times 10^{-9}$ & $1.226 \times 10^{-7}$ \\
\hline
\end{tabular}

\section{1.b. Longitudinal and Hall Resistivities for Constant Magnetic Field}

Within the framework of present theory, the numerical calculations of the Hall and longitudinal resistivities when the applied magnetic field is kept at a constant value $B=2.24$ Tesla are given in Table 2. It is shown that as

Table 2. $\rho_{x y}$ and $\rho_{x x}$ versus Temperature for $\mathrm{YBa}_{2} \mathrm{Cu}_{3} \mathrm{O}_{7-\delta}$ High- $T_{c}$ Superconducting Bulk Materials at $B=2.24$ Tesla

\begin{tabular}{|c|c|c|}
\hline $\boldsymbol{T}(\boldsymbol{K})$ & $\boldsymbol{\rho}_{x y}(\boldsymbol{\Omega} \boldsymbol{m})$ & $\boldsymbol{\rho}_{x x}(\boldsymbol{\Omega} \boldsymbol{m})$ \\
\hline \hline 91.6 & $6.96 \times 10^{-10}$ & $1.878 \times 10^{-6}$ \\
\hline 91.3 & $-1.82 \times 10^{-11}$ & $1.396 \times 10^{-6}$ \\
\hline 91 & $-2.491 \times 10^{-9}$ & $1.094 \times 10^{-6}$ \\
\hline 90 & $-4.199 \times 10^{-9}$ & $7.028 \times 10^{-7}$ \\
\hline 89 & $-4.722 \times 10^{-9}$ & $5.22 \times 10^{-7}$ \\
\hline 88 & $-3.081 \times 10^{-9}$ & $4.91 \times 10^{-7}$ \\
\hline
\end{tabular}

temperature decreasing, $\rho_{x y}$ initially decreases, crossing over from positive to negative near $91.3 \mathrm{~K}$, after reaching a minimum at $89 \mathrm{~K}$, then increases again; while $\rho_{x x}$ decreases monotonically. These results are in agreement with the experimental plotting for $\rho_{x y}$ and $\rho_{x x}$ versus temperature on $\mathrm{YBa}_{2} \mathrm{Cu}_{3} \mathrm{O}_{7-\delta}$ high- $T_{c}$ bulk materials [17]. In arriving at the above results, the following approximate data have been used: $R=2 \times 10^{-8} \mathrm{~m}, L=10^{-6} \mathrm{~m}, J=10^{6} \mathrm{~A} / \mathrm{m}^{2}, T_{c}=92 \mathrm{~K}$,
$v_{T}=10^{3} \mathrm{~m} / \mathrm{sec}, \quad \bar{v}=10^{11} \mathrm{sec}^{-1}, \quad \bar{\alpha}=5.59 \times 10^{-5} T^{-1 / 2}$, $\exp \left(-U / k_{B} T\right)=2.07 \times 10^{-2}$,

$\left(\beta^{C}(T=91.6) / \bar{V}\right)^{1 / 2}=2.178 \times 10^{6} \mathrm{~N} / \mathrm{m}^{3}$,

$\left(\beta^{C}(91.3) / \bar{V}\right)^{1 / 2}=2.194 \times 10^{6} \mathrm{~N} / \mathrm{m}^{3}$,

$\left(\beta^{C}(91) / \bar{V}\right)^{1 / 2}=2.204 \times 10^{6} \mathrm{~N} / \mathrm{m}^{3}$, $\left(\beta^{C}(90) / \bar{V}\right)^{1 / 2}=2.217 \times 10^{6} \mathrm{~N} / \mathrm{m}^{3}$,

$\left(\beta^{C}(89) / \bar{V}\right)^{1 / 2}=2.223 \times 10^{6} \mathrm{~N} / \mathrm{m}^{3}$, and $\left(\beta^{C}(88) / \bar{V}\right)^{1 / 2}=2.224 \times 10^{6} \mathrm{~N} / \mathrm{m}^{3}$.

\subsection{Induced Longitudinal and Hall Resistivities for Type-II Superconducting Films}

Now let us turn our attention to type-II superconducting films, the volume $\bar{V}$ of the vortex bundle is therefore expressed by $\bar{V}=\pi R^{2} d$, with $R$ the transverse size of the vortex bundle and $d$ the thickness of the film. The longitudinal and Hall resistivities of Eqs. (29) and (30) can now be described as

$$
\begin{aligned}
& \rho_{x x}= \frac{\bar{v} \sqrt{\Phi_{0}} \sqrt{B}}{J \sqrt{\pi} \sqrt{T}} \exp \left(\frac{-U}{k_{B} T}\right)\left[\frac{2 \pi R^{3} d}{k_{B}}\right]\left[J B-\left(\frac{\beta^{C}(T, B)}{\bar{V}}\right)^{\frac{1}{2}}\right] \\
& \rho_{x y}= \frac{-\bar{\nu} \sqrt{\Phi_{0}} \sqrt{B}}{J \sqrt{\pi} \sqrt{T}} \exp \left(\frac{-U}{k_{B} T}\right)\left[\frac{2 \pi R^{3} d}{k_{B}}\right]\left[\left(\frac{\beta^{C}(T, B)}{\bar{V}}\right)^{\frac{1}{2}}\right. \\
&\left.\bar{\alpha} \sqrt{\frac{T}{T_{c}-T}}-J B \frac{\left|v_{b y}\right|}{v_{T}}\right]
\end{aligned}
$$

respectively, with $\left|v_{b y}\right|=J \rho_{x x} / B$.

\section{2.a. Longitudinal and Hall Resistivities for Constant Temperature}

Under the framework of the present theory, the numerical calculations of $\rho_{x y}$ and $\rho_{x x}$ as functions of applied magnetic field in Tesla, when temperature is kept at a constant value $T=4.5 K$, are given in Table 3 . It is shown that as the applied magnetic field decreasing, $\rho_{x y}$ initially decreases, crossing over from positive to negative between 7.25 and 7 Tesla, after reaching a minimum at 5.75 Tesla, then increases again; while $\rho_{x x}$ decreases monotonically. These results are in agreement with the experimental plotting for $\rho_{x y}$ and $\rho_{x x}$ versus applied magnetic field on $\mathrm{Mo}_{3} \mathrm{Si}$ conventional low$T_{c}$ superconducting films [18]. In obtaining the above results, the following approximate data have been employed: $R=2 \times 10^{-8} \mathrm{~m}, \quad d=5 \times 10^{-8} \mathrm{~m}, \quad J=1.5 \times 10^{5} \mathrm{~A} / \mathrm{m}^{2}$, $T_{c}=7.5 \mathrm{~K}, \quad v_{T}=30 \mathrm{~m} / \mathrm{sec}, \quad \bar{\alpha}=1.0449 \times 10^{-3} T^{-1 / 2}$, 


$$
\begin{aligned}
& \bar{V}=10^{11} \mathrm{sec}^{-1}, \\
& \exp \left(-U / k_{B} T\right)=3.0899 \times 10^{-4} \\
& \left(\beta^{C}(B=7.5) / \bar{V}\right)^{1 / 2}=4.5772 \times 10^{5} \mathrm{~N} / \mathrm{m}^{3} \\
& \left(\beta^{C}(7.25) / \bar{V}\right)^{1 / 2}=4.4737 \times 10^{5} \mathrm{~N} / \mathrm{m}^{3} \\
& \left(\beta^{C}(7) / \bar{V}\right)^{1 / 2}=4.4324 \times 10^{5} \mathrm{~N} / \mathrm{m}^{3} \\
& \left(\beta^{C}(6.75) / \bar{V}\right)^{1 / 2}=4.3964 \times 10^{5} \mathrm{~N} / \mathrm{m}^{3} \\
& \left(\beta^{C}(6.5) / \bar{V}\right)^{1 / 2}=4.3483 \times 10^{5} \mathrm{~N} / \mathrm{m}^{3} \\
& \left(\beta^{C}(6.25) / \bar{V}\right)^{1 / 2}=4.272 \times 10^{5} \mathrm{~N} / \mathrm{m}^{3} \\
& \left(\beta^{C}(6) / \bar{V}\right)^{1 / 2}=4.0516 \times 10^{5} \mathrm{~N} / \mathrm{m}^{3} \\
& \left(\beta^{C}(5.75) / \bar{V}\right)^{1 / 2}=3.7418 \times 10^{5} \mathrm{~N} / \mathrm{m}^{3} \\
& \text { and }\left(\beta^{C}(5.5) / \bar{V}\right)^{1 / 2}=3.335 \times 10^{5} \mathrm{~N} / \mathrm{m}^{3}
\end{aligned}
$$

Table 3. $\rho_{x y}$ and $\rho_{x x}$ versus Applied Magnetic Field in Tesla for $\mathrm{Mo}_{3} \mathrm{Si}$ Conventional Low- $\mathrm{T}_{c}$ Superconducting Films at $T=4.5 K$

\begin{tabular}{|c|c|c|}
\hline $\boldsymbol{B}(\boldsymbol{T})$ & $\rho_{x y}(\boldsymbol{\Omega} \boldsymbol{m})$ & $\rho_{x x}(\boldsymbol{\Omega} \boldsymbol{m})$ \\
\hline \hline 7.5 & $4.3399 \times 10^{-11}$ & $8.2782 \times 10^{-7}$ \\
\hline 7.25 & $1.5804 \times 10^{-11}$ & $7.8079 \times 10^{-7}$ \\
\hline 7.0 & $-2.6037 \times 10^{-11}$ & $7.2721 \times 10^{-7}$ \\
\hline 6.75 & $-6.7189 \times 10^{-11}$ & $6.742 \times 10^{-7}$ \\
\hline 6.5 & $-1.023 \times 10^{-10}$ & $6.24 \times 10^{-7}$ \\
\hline 6.25 & $-1.283 \times 10^{-10}$ & $5.78 \times 10^{-7}$ \\
\hline 6.0 & $-1.1842 \times 10^{-10}$ & $5.492 \times 10^{-7}$ \\
\hline 5.75 & $-8.8118 \times 10^{-11}$ & $5.3044 \times 10^{-7}$ \\
\hline 5.5 & $-3.752 \times 10^{-11}$ & $5.2209 \times 10^{-7}$ \\
\hline
\end{tabular}

\section{2.b. Longitudinal and Hall Resistivities for Constant Magnetic Field}

The results of numerical calculations for $\rho_{x y}$ and $\rho_{x x}$ as functions of temperature when the applied magnetic field is kept at a constant value $B=2$ Tesla are given in Table 4. It is shown that as temperature decreasing, $\rho_{x y}$ initially decreases, crossing over from positive to negative near 100 $\mathrm{K}$, after reaching a minimum at $96 \mathrm{~K}$, then increases, crossing over back from negative to positive near $88 \mathrm{~K}$, reaching a local maximum at about $78 \mathrm{~K}$, then decreases again; while $\rho_{x x}$ decreases monotonically. These results are in agreement with the experimental plotting for $\rho_{x y}$ and $\rho_{x x}$ versus temperature on $\mathrm{Tl}_{2} \mathrm{Ba}_{2} \mathrm{Cu}_{2} \mathrm{O}_{8}$ high- $T_{c}$ superconducting films [19]. In obtaining the above results, the following approximate data have been used:

$$
\begin{aligned}
& R=2 \times 10^{-8} \mathrm{~m}, \quad d=10^{-6} \mathrm{~m}, \quad J=10^{7} \mathrm{~A} / \mathrm{m}^{2}, \quad T_{c}=104 \mathrm{~K}, \\
& v_{T}=10^{2} \mathrm{~m} / \mathrm{sec}, \bar{v}=10^{11} \mathrm{sec}^{-1}, \\
& \bar{\alpha}=1.12 \times 10^{-4} T^{-1 / 2}, \quad \exp \left(-U / k_{B} T\right)=8.3199 \times 10^{-5} \\
& \left(\beta^{C}(T=102) / \bar{V}\right)^{1 / 2}=1.8464 \times 10^{7} \mathrm{~N} / \mathrm{m}^{3}, \\
& \left(\beta^{C}(100) / \bar{V}\right)^{1 / 2}=1.9031 \times 10^{7} \mathrm{~N} / \mathrm{m}^{3} \\
& \left(\beta^{C}(98) / \bar{V}\right)^{1 / 2}=1.93267 \times 10^{7} \mathrm{~N} / \mathrm{m}^{3} \\
& \left(\beta^{C}(96) / \bar{V}\right)^{1 / 2}=1.9512 \times 10^{7} \mathrm{~N} / \mathrm{m}^{3} \\
& \left(\beta^{C}(92) / \bar{V}\right)^{1 / 2}=1.955 \times 10^{7} \mathrm{~N} / \mathrm{m}^{3} \\
& \left(\beta^{C}(88) / \bar{V}\right)^{1 / 2}=1.95618 \times 10^{7} \mathrm{~N} / \mathrm{m}^{3} \\
& \left(\beta^{C}(84) / \bar{V}\right)^{1 / 2}=1.9588 \times 10^{7} \mathrm{~N} / \mathrm{m}^{3} \\
& \left(\beta^{C}(78) / \bar{V}\right)^{1 / 2}=1.961069 \times 10^{7} \mathrm{~N} / \mathrm{m}^{3} \\
& \text { and }\left(\beta^{C}(76) / \bar{V}\right)^{1 / 2}=1.9631 \times 10^{7} \mathrm{~N} / \mathrm{m}^{3} .
\end{aligned}
$$

Table 4. $\rho_{x y}$ and $\rho_{x x}$ as Functions of Temperature for $\mathrm{Tl}_{2} \mathrm{Ba}_{2} \mathrm{Cu}_{2} \mathrm{O}_{8} \mathrm{High}-\mathrm{T}_{c} \quad$ Superconducting Thin Films at $B=2$ Tesla

\begin{tabular}{|c|c|c|}
\hline $\boldsymbol{T}(\boldsymbol{K})$ & $\boldsymbol{\rho}_{x y}(\boldsymbol{\Omega} \boldsymbol{m})$ & $\boldsymbol{\rho}_{x x}(\boldsymbol{\Omega} \boldsymbol{m})$ \\
\hline \hline 102 & $2.1346 \times 10^{-11}$ & $1.6728 \times 10^{-8}$ \\
\hline 100 & $-3.69 \times 10^{-19}$ & $1.0657 \times 10^{-8}$ \\
\hline 98 & $-1.4607 \times 10^{-11}$ & $7.4819 \times 10^{-9}$ \\
\hline 96 & $-2.3518 \times 10^{-11}$ & $5.4754 \times 10^{-9}$ \\
\hline 92 & $-1.0344 \times 10^{-11}$ & $5.1609 \times 10^{-9}$ \\
\hline 88 & $5.9409 \times 10^{-19}$ & $5.1382 \times 10^{-9}$ \\
\hline 84 & $5.46 \times 10^{-12}$ & $4.951 \times 10^{-9}$ \\
\hline 78 & $1.3011 \times 10^{-11}$ & $4.8488 \times 10^{-9}$ \\
\hline 76 & $1.2967 \times 10^{-11}$ & $4.65 \times 10^{-9}$ \\
\hline
\end{tabular}

\section{CONCLUSION}

The theory of the thermally activated vortex bundles flow over the directional-dependent potential barrier induced by the Magnus force, random collective pinning random force, and strong pinning force inside the vortex bundles for type-II superconductors is developed. The coherent oscillation frequency and the mean direction of the random collective pinning force of the vortex bundle are evaluated. The bundle flow velocity is obtained. Finally, the longitudinal and Hall resistivities induced by the bundle flow are calculated for type-II superconducting bulk materials as well as thin films. The results are in agreement with the experiments.

\section{ACKNOWLEDGEMENT}

The authors would like to thank Professors E. H. Brandt for helpful discussions and suggestions. 


\section{REFERENCES}

[1] Abrikosov AA. On the magnetic properties of superconductors of the second group. J Exp Theor Phys 1957; 5: 1174-82.

[2] Kleiner WM, Roth LM, Autler SH. Bulk solution of GinzburgLandau equations for type-II superconductors: upper critical field region. Phys Rev 1964; 133: A1226-7.

[3] Anderson PW. Theory of flux creep in hard superconductors. Phys Rev Lett 1962; 9: 309-11.

[4] Campbell AM, Evetts JE. Flux vortices and transport current in type-II superconductors. Adv Phys 1972; 21: 199-428.

[5] Brandt EH. The flux-line lattice in superconductors. Rep Prog Phys 1995; 58: 1465-1594.

[6] Larkin AI, Ovchinnikov Yu N. Pinning in type II superconductors. J Low Temp Phys 1979; 43: 409-28.

[7] Chen WY, Chou MJ. Theories of peak effect and anomalous Hall effect for cuprate superconductors. In: Courtlandt KN, Ed. Superconducting cuprates: properties, preparation and applications, New York: Nova Science Publishers 2009; pp. 213-34.

[8] Chen WY, Chou MJ. Theories of peak effect and anomalous Hall effect in superconducting MgB2. In: Suzuki S, Fukuda K, Eds. Magnesium Diboride (MgB2) Superconductor Research, New York: Nova Science Publishers 2009; pp. 153-74.

[9] Chen WY, Chou MJ. The quasiorder-disorder phase transition and the peak effect in type-II conventional and high-Tc superconductor. Supercond Sci Tech 2006; 19: 237-41.

[10] Chen WY, Chou MJ, Feng S. Quasiorder-disorder phase transition and induced peak effect in type-II superconductors. Phys C 2007; 460-462: 1236-7.

[11] Chen WY, Chou MJ, Feng S. The feature of quantum and thermal fluctuations on collective pinning and critical current in superconducting film. Phys Lett A 2003; 316: 261-4.
[12] Chen WY, Chou MJ. Fluctuation-induced attraction of vortices in anisotropic superconductors. Phys Lett A 2001; 280: 371-5.

[13] Brandt EH. Flux line lattice in high-Tc superconductors: anisotropy, elasticity, fluctuation, thermal depinning, AC penetration and susceptibility. Phys C 1992; 195: 1-27.

[14] Coffey MW, Clem JR. Vortex inertial mass for a discrete type-II superconductor. Phys Rev B 1991; 44: 6903-8.

[15] Chou MJ, Chen WY. Vortex-pair production in quenched disorder superconducting film. Phys Lett A 2004; 332: 405-11.

[16] Chen WY, Chou MJ, Feng S. The effect of collective pinning on topological order-disorder phase transition in type-II superconducting films. Phys Lett A 2005; 342: 129-33.

[17] Galffy M, Zirngiebl E. Hall-effect of bulk $\mathrm{YBa}_{2} \mathrm{Cu}_{3} \mathrm{O}_{7-\delta}$. Solid State Commun 1988; 68: 929-33.

[18] Smith AW, Clinton TW, Tsuei CC, et al. Sign reversal of the Hall resistivity in amorphous Mo Si . Phys Rev B 1994; 49: 12927-30.

[19] Hagen SJ, Lobb CJ, Greene RL, et al. Flux-flow Hall effect in superconducting $\mathrm{Tl}_{2} \mathrm{Ba}_{2} \mathrm{Cu}_{2} \mathrm{O}_{8}$ films. Phys Rev B 1991; 43: 6246.

[20] Yang HC, Wang LM, Horng HE. Anomalous Hall effects of $\mathrm{Md}_{07} \mathrm{Sr}_{03} \mathrm{MnO}_{8}$ : Evidence of the Berry phase effect. Phys Rev B 2001; 64: 174415.

[21] Yang HC, Wang LM, Horng HE. Characteristics of flux pinning in $\mathrm{YBa}_{2} \mathrm{Cu}_{3} \mathrm{O} / \mathrm{Pr} \mathrm{Ba}_{2} \mathrm{Cu}_{3} \mathrm{O}$ superlattices. Phys Rev B 1999; 59: 8956.

[22] Zheng H, Zhu SY, Chen WY. Quantum phonons and the Peierls transition temperature. Phys Rev B 2001; 65: 014304.

[23] Kes PH, Tsuei CC. Collective-flux-pinning phenomena in amorphous superconductors. Phys Rev Lett 1981; 47: 1930.

[24] Kes PH, Tsuei CC. Two-dimensional collective flux pinning, defects, and structural relaxation in amorphous superconducting films. Phys Rev B 1983; 28: 5126.

(C) Wei Yeu Chen; Licensee Bentham Open.

This is an open access article licensed under the terms of the Creative Commons Attribution Non-Commercial License (http://creativecommons.org/ licenses/by-nc/3.0/), which permits unrestricted, non-commercial use, distribution and reproduction in any medium, provided the work is properly cited. 